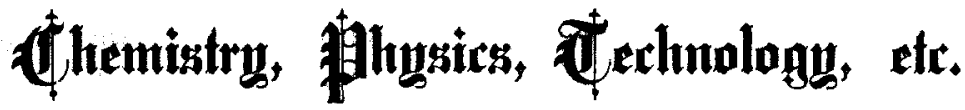

\section{ON THE EFFECT OF ACID ON THE INTERIOR OF IRON WIRE.*}

\author{
By Professor Osborne Reynolds, M. A.
}

It will be remembered that at a previous meeting of this society, Mr. Johnson exhibited some iron and steel wire in which he had observed some very singular effects produced by the action of sulphuric acid. In the first place the nature of the wire was changed in a marked manner, for although it was soft charcoal wire it had become short and brittle; the weight of the wire was increased; and what was the most remarkable effect of all was that when the wire was broken, and the face of the fracture wetted with the mouth, it frothed up as if the water acted as a powerful acid. These effects, however, all passed off if the wire were allowed to remain exposed to the air for some days, and if it were warmed before the fire, they passed off in a few hours.

By Mr. Johnson's permission, I took possession of one of these pieces of wire and subjected it to a farther examination, and from the result of that examination $I$ was led to what appears to me to be a complete explanation of the phenomena. I observed that when I broke a short piece from the end of the wire the two faces of the fracture behaved very differently-that on the long piece frothed when wetted and continued to do so for some seconds, while that on the short piece would hardly show any signs of froth at all. This seemed to imply that the gas which caused the froth came from a considerable depth below the surface of the wire, and was not generated on the freshly exposed face. This view was confirmed when on substituting oil for water I found the froth just the same. These observations led me to conclude that the effect was due to hydrogen, and not to acid, as Mr. Johnson appeared to think, having entered into combination with the iron during its immersion in the acid, which hydrogen gradually passed off when the iron was exposed. 'It

* A paper read before the Manchester Literary and Philosophical Society. Re printed from the London Engineer. 
was obvious, however, that this conclusion was capable of being further tested. It was clearly possible to ascertain whether or not the gas was hydrogen, and whether hydrogen penetrated iron when under the action of acid. With a view to do this I made the following experiments.

First, however, I would mention that after twenty-four hours I examined what remained of the wire, when $I$ found that all appearance of frothing had vanished and the wire had recovered its ductility, so much so that it would now bend backwards and forwards two or three times without breaking, whereas on the previous evening a single bend had sufficed to break it. I then obtained a piece of wrought iron gas pipe, 6 inches long and $\frac{5}{8}$ inch external diameter, and rather more than 1-16 inch thick; I had this cleaned in a lathe both inside and outside; over one end I soldered a piece of copper so as to stop it, and the other I connected with a piece of glass tube by means of india-rubber tubes. I then filled both the glass and iron tubes with olive oil and immersed the iron tube in diluted sulphuric acid which had been mixed for some time and was cold. Under this arrangement any hydrogen which came from the inside of the glass tube must have passed through the iron. After the iron had been in acid about five minutes small bubbles began to pass up the glass tube. These were caught at the top and were subsequently burnt and proted to be hydrogen. At first, however, they came off but very slowly, and it was several hours before I had collected enough to burn. With a view to increase the speed I changed the acid several times without much effect until I happened to use some acid which had only just been diluted and was warm; then the gas came off twenty or thirty times as fast as it had previously done. I then put a lamp under the bath and measured the rate at which the gas came off, and I found that when the acid was on the point of boiling as much hydrogen was given off in five seconds as had previously come off in ten minutes, and the rate was maintained in both cases for several hours. After having been in acid some time the tube was taken out, well washed with cold water and soap so as to remove all trace of the acid; it was then plunged into a bath of hot water, upon which gas came off so. rapidly from both the outside and inside of the tube as to give the appearance of the action of strong acid. This action lasted for some time, but gradually diminished. It could be stopped at any time by the substitution of cold water in place of the hot, and it was renewed 
again after several hours by again putting the tube in hot water. The volume of hydrogen which was thus given off by the tube after it had been taken out of hot acid was about equal to the volume of the iron. At the time I made these experiments I was not aware that there had been any previous experiments on the subject; but I subsequently found, on referring to Watts' "Dictionary of Chemistry," that Cailletet had in 1868 discovered that hydrogen would pass into an iron vessel immersed in sulphuric acid. See Comp. Rend. lxvi., 847: The facts thus established appear to afford a complete explanation of the effects observed by Mr. Johnson.

In the first place, with regard to the temporary character of the effect, it appears that the hydrogen leaves the iron. slowly even at ordinary temperatures-so much so that after two or three days' exposure I found no hydrogen given off when the tube was immersed in hot water. With regard to the effect of warming the wire-at the temperature of boiling the hydrogen passed off 120 times as fast as at the temperature of $60 \mathrm{deg}$. Also when the saturated iron was plunged into warm water the gas passed off as if the iron had been plunged into strong acid; so that we can easily understand how the hydrogen would pass off from the wire quickly when warm, although it would take long to do so at the ordinary temperatures. With regard to the frothing of the wire when broken and wetted, this was not due, as at first sight it appeared to be, simply to the exposure of the interior of the wire, but was due to warmth caused in the wire by the act of breaking. This was proved by the fact that the froth appeared on the sides of the wire in the immediate neighborhood of the fracture, when these were wetted, as well as the end; and by simply bending the wire it could be made to froth at the point where it was bent. As to the effect on the nature and strength of the iron, I cannot add anything to what Mr. Johnson has already observed. The question, however, appears to be one of very considerable importance, both philosophically and in connection with the use of iron in the construction of ships and boilers. If, as is probable, the saturation of iron with hydrogen takes place whenever oxidation goes on in water, then the iron of boilers and ships may at times be changed in character and rendered brittle in the same manner as Mr. Johnson's wire, and this, whether it can be prevented or not, is at least an important point to know, and would repay a further investigation of the subject. 